\title{
Caracterização físico-química de frutos de maracujazeiro amarelo provenientes da irradiação com raios gama
}

\author{
Physico-chemical characterization of fruits of passion fruit obtained from gamma irradiation \\ Patrícia Silva Flores ${ }^{\mathrm{I}}$ Danielle Fabíola Pereira da Silva ${ }^{\mathrm{II}}$ Cláudio Horst Bruckner ${ }^{\mathrm{II}}$ \\ Sílvia Paula de Oliveira ${ }^{\mathrm{II}}$ Luiz Carlos Chamhum Salomão ${ }^{\mathrm{II}}$
}

\begin{abstract}
A indução de mutações tem sido utilizada em
de melhoramento de algumas culturas RESUMO economicamente importantes, como ferramenta para complementar as técnicas de melhoramento convencional. Em estudos prévios, observou-se que, através da indução de mutações com irradiações com raios gama, foi possível obter plantas de maracujazeiro amarelo resistentes a fusariose, uma das principais doenças que ataca a cultura. Porém, estudos sobre caracteres agronômicos relacionados com a qualidade dos frutos são fundamentais para avaliação desses genótipos resistentes. Neste trabalho, foram analisadas as características físicas e químicas dos frutos de plantas de Passiflora edulis Sims provenientes da irradiação com raios gama. Os valores obtidos com as análises foram muito próximos àqueles encontrados na literatura relacionada com a caracterização físico-química de frutos de maracujazeiro amarelo. Porém, será necessário o melhoramento para redução da espessura da casca.
\end{abstract}

Palavras-chave: Passiflora edulis, qualidade do fruto, indução de mutações.

\section{ABSTRACT}

The induction of mutations has been used in breeding programs of some economically important crops as a tool to complement conventional breeding techniques. In previous studies, it was found that by inducing mutations with gamma rays it was possible to obtain yellow passion fruit plants resistant to fusarium wilt, a major disease of the culture. However, studies on agronomic traits related to fruit quality are necessary to evaluating these resistant genotypes. In this study the physical and chemical characteristics of fruits of Passiflora edulis Sims plants obtained from gamma irradiation were analyzed. The values found were similar to those in the literature related to the physico-chemical characterization of fruits of yellow passion fruit. However, a program breeding for the reducing of the skin thickness of the fruits will be necessary.

Key words: Passiflora edulis, fruit quality, induction of mutations.

A indução de mutações é uma ferramenta útil no melhoramento genético de plantas, sendo utilizada no melhoramento de culturas importantes como trigo, arroz, cevada, algodão, amendoim e feijãocaupi. As principais vantagens da indução de mutações no melhoramento genético incluem a criação de alelos inexistentes no pool gênico de uma espécie e a possibilidade de aumentar uma ou duas características agronômicas de uma variedade superior, sem mudar o restante do genótipo (AL-QURAINY \& KHAN, 2009).

Além da busca por características de resistência a patógenos, estudos sobre caracteres agronômicos relacionados com a qualidade dos frutos são fundamentais para avaliação de genótipos superiores de maracujazeiro amarelo. Segundo FERREIRA et al. (2010), caracteres como peso de fruto, comprimento de fruto e diâmetro equatorial do fruto são importantes em programas de melhoramento genético do maracujazeiro para obtenção de genótipos com características comerciais para mercado in natura. Para a produção de maracujá destinado à indústria de

IEmpresa Brasileira de Pesquisa Agropecuária - Acre (Embrapa Acre), BR-364, km 14, CP 321, 69908-970, Rio Branco, Acre, Brasil. E-mail: patricia.flores@cpafac.embrapa.br. Autor para correspondência.

"Departamento de Fitotecnia, Universidade Federal de Viçosa (UFV), Viçosa, MG, Brasil. 
sucos, o elevado teor de ácido no suco é uma característica importante ao processamento por diminuir a adição de acidulante durante o processo de concentração dos sucos. Segundo NAGATO et al. (2003), sucos de maracujá com pH de 2,8 a 3,1, sólidos solúveis totais de 11,4 a $15,3^{\circ}$ Brix, acidez titulável de 2,7 a $3,9 \mathrm{~g}$ de ácido cítrico $100 \mathrm{~mL}^{-1}$ e relação brix/acidez de 3,5 a 4,7, apresentam maior aceitabilidade sensorial.

O presente trabalho objetivou analisar as características físicas e químicas dos frutos de plantas de Passiflora edulis f flavicarpa irradiadas com raios gama.

Foram utilizadas plantas mutantes de $\boldsymbol{P}$. edulis f flavicarpa resistentes ao fungo Fusarium oxysporum f. sp. passiflorae, com dois anos de idade. Para a obtenção dos mutantes resistentes, segmentos nodais de maracujazeiro amarelo foram tratados com raios gama de 20Gy e submetidos ao primeiro ciclo de seleção in vitro em meio de cultura contendo $40 \%$ de filtrado da cultura fúngica e, em seguida, a um segundo ciclo em meio contendo $50 \%$ do filtrado. Após a aclimatização, as plantas sobreviventes foram transferidas para vasos contendo areia autoclavada e inoculadas com a suspensão do esporo do fungo $\left(4 \times 10^{7}\right.$ esporos $\left.\mathrm{mL}^{-1}\right)$. As plantas sobreviventes foram transferidas para o campo, em área com histórico de ocorrência de fusariose, juntamente com plantas não irradiadas, utilizadas como controle, sendo conduzidas em espaldeira com um fio de arame e irrigadas através de sistema de aspersores na área experimental do Setor de Fruticultura da Universidade Federal de Viçosa.

Para as análises das características físicas e químicas dos frutos, foram coletados 10 frutos maduros por planta, com 63 dias após a antese, quando apresentavam coloração de casca totalmente amarela (VIANNA-SILVA, 2010).

Os frutos foram obtidos de polinização natural e, depois de atingirem cerca de $5 \mathrm{~cm}$ de comprimento, foram cobertos com sacos de organza $(20 \times 20 \mathrm{~cm})$ para evitar o ataque de pragas. Após a colheita, foram levados para o Laboratório de Análise de Frutas da Universidade Federal de Viçosa, onde foram realizadas as análises químicas e físicas.

Os frutos e suas partes foram pesados em balança eletrônica de precisão de $0,1 \mathrm{~g}$. A massa da polpa foi determinada por subtração (massa dos frutos massa das cascas). O cálculo das massas da polpa e da casca foi feito em relação à massa total dos frutos. $\mathrm{O}$ comprimento e diâmetro dos frutos foram determinados através de paquímetro.

A cor da polpa foi analisada com colorímetro MINOLTACR-10, com base nos valores de L, $\mathrm{C} \mathrm{e} \mathrm{h}{ }^{\circ}$. A análise do teor de sólidos solúveis, acidez titulável e teor de vitamina $\mathrm{C}$ da polpa foram determinados a partir de alíquotas da polpa de três frutos. Os sólidos solúveis foram determinados através de três leituras na faixa de 0 a $32^{\circ}$ Brix em refratômetro portátil Atago N1. A acidez titulável e o teor de vitamina $\mathrm{C}$ do suco foram avaliados conforme técnicas desenvolvidas pela AOAC (1997).

A massa dos frutos variou de 102,5 a 202,06g. Segundo CAVICHIOLF et al. (2008), os consumidores preferem frutos superiores a $170 \mathrm{~g}$. Nesse sentido, os genótipos mutantes de número 10 e 3121 se destacaram, ficando dentro dos padrões de comercialização.

O diâmetro dos frutos variou de 60,08 a $76,70 \mathrm{~mm}$ (Tabela 1). Os valores são semelhantes à variação de $64,5 \mathrm{a} 77,7 \mathrm{~mm}$ apresentada por RODRIGUES

Tabela 1 - Caracterização física e química de frutos de plantas mutantes de maracujazeiro amarelo (Passiflora edulis $\mathrm{f}$. flavicarpa). MF=massa do fruto, $\mathrm{DF}=$ diâmetro do fruto, $\mathrm{CF}=$ comprimento do fruto, $\mathrm{EC}=$ espessura da casca, $\mathrm{MC}=$ massa da casca, $\mathrm{MP}=\mathrm{massa}$ da polpa, $\mathrm{RC}=$ rendimento de casca, $\mathrm{RS}=$ rendimento de suco.

\begin{tabular}{|c|c|c|c|c|c|c|c|c|c|c|c|c|c|c|c|}
\hline \multirow{2}{*}{ Planta } & \multirow{2}{*}{ MF (g) } & \multirow{2}{*}{$\mathrm{DF}(\mathrm{mm})$} & \multirow{2}{*}{$\begin{array}{c}\mathrm{CF} \\
(\mathrm{mm})\end{array}$} & \multirow{2}{*}{$\begin{array}{l}\mathrm{CF} / \\
\mathrm{DF}\end{array}$} & \multirow{2}{*}{$\begin{array}{c}\mathrm{EC} \\
(\mathrm{mm})\end{array}$} & \multirow{2}{*}{$\mathrm{MC}(\mathrm{mg})$} & \multirow{2}{*}{ MP (g) } & \multirow{2}{*}{ RS (\%) } & \multirow{2}{*}{$\begin{array}{c}\text { Acidez } \\
\text { titulável* }\end{array}$} & \multirow{2}{*}{$\begin{array}{l}\text { Sólidos } \\
\text { Solúveis } \\
\text { ( }{ }^{\circ} \text { Brix) }\end{array}$} & \multirow{2}{*}{$\begin{array}{l}\text { sólidos } \\
\text { solúveis/ } \\
\text { acidez } \\
\text { titulável }\end{array}$} & \multirow{2}{*}{$\begin{array}{c}\text { Vitamina } \\
\mathrm{C}^{* *}\end{array}$} & \multicolumn{3}{|c|}{-----Coloração da polpa---- } \\
\hline & & & & & & & & & & & & & $\mathrm{L}$ & $\mathrm{C}$ & $h^{o}$ \\
\hline 10 & $202,06 \mathrm{a}$ & $76,70 \mathrm{a}$ & $85,88^{\mathrm{ns}}$ & 1,11 & $8,32 \mathrm{a}$ & $107,90 \mathrm{a}$ & $94,16 a$ & $41,37 \mathrm{a}$ & $4,0 \mathrm{bc}$ & $14,8 \mathrm{bcd}$ & $3,7 b$ & $27,7 \mathrm{bc}$ & $45,37 \mathrm{bc}$ & $19,94 \mathrm{abc}$ & $73,90 \mathrm{c}$ \\
\hline 14 & $149,82 \mathrm{ab}$ & $69,36 \mathrm{abc}$ & $79,95^{\mathrm{ns}}$ & 1,15 & $6,33 b$ & $80,88 \mathrm{abc}$ & $68,94 \mathrm{ab}$ & $37,90 \mathrm{ab}$ & $4,2 b$ & $14,9 \mathrm{bc}$ & $3,6 b$ & $20,6 \mathrm{~d}$ & $45,50 \mathrm{bc}$ & $21,02 \mathrm{abc}$ & $78,44 a b c$ \\
\hline 19 & $135,01 \mathrm{ab}$ & $71,72 \mathrm{ab}$ & $84,97^{\mathrm{ns}}$ & 1,18 & $5,85 \mathrm{~b}$ & $81,33 \mathrm{abc}$ & $53,68 \mathrm{ab}$ & $34,00 \mathrm{ab}$ & $4,4 \mathrm{a}$ & $14,3 \mathrm{~d}$ & $3,2 \mathrm{c}$ & $28,2 b$ & $45,60 \mathrm{bc}$ & $17,75 \mathrm{bc}$ & $77,15 b c$ \\
\hline 23 & $156,48 \mathrm{ab}$ & $70,23 \mathrm{ab}$ & $78,44^{\mathrm{ns}}$ & 1,12 & $6,51 b$ & $82,58 \mathrm{abc}$ & $73,90 \mathrm{ab}$ & $40,14 \mathrm{a}$ & $3,5 \mathrm{f}$ & $13,1 \mathrm{e}$ & $3,8 b$ & $23,2 \mathrm{~cd}$ & $46,08 \mathrm{bc}$ & $22,03 \mathrm{abc}$ & $75,88 \mathrm{bc}$ \\
\hline 24 & $102,51 \mathrm{~b}$ & $60,08 \mathrm{c}$ & $75,29^{\mathrm{ns}}$ & 1,25 & $6,36 b$ & $58,87 \mathrm{c}$ & $43,65 b$ & $35,70 \mathrm{ab}$ & $3,9 \mathrm{~cd}$ & $14,5 \mathrm{~cd}$ & $3,7 b$ & $34,9 \mathrm{a}$ & $44,68 b c$ & $19,20 \mathrm{abc}$ & $82,35 \mathrm{ab}$ \\
\hline 27 & $127,07 \mathrm{~b}$ & $64,75 b c$ & $75,73^{\mathrm{ns}}$ & 1,21 & $6,65 b$ & $71,98 \mathrm{bc}$ & $55,09 \mathrm{ab}$ & $34,01 \mathrm{ab}$ & $3,8 \mathrm{de}$ & $15,5 \mathrm{ab}$ & $4,1 \mathrm{a}$ & $25,6 b c$ & $47,68 \mathrm{ab}$ & $24,89 \mathrm{ab}$ & $77,85 \mathrm{abc}$ \\
\hline 318 & $123,35 b$ & $67,73 \mathrm{abc}$ & $78,62^{\mathrm{ns}}$ & 1,16 & $6,76 b$ & $77,82 \mathrm{bc}$ & $45,53 \mathrm{~b}$ & $29,17 b$ & $3,7 \mathrm{e}$ & $13,6 \mathrm{e}$ & $3,7 b$ & $34,7 \mathrm{a}$ & $51,42 \mathrm{a}$ & $27,85 \mathrm{a}$ & $79,61 \mathrm{abc}$ \\
\hline 3121 & $169,48 \mathrm{ab}$ & $72,66 \mathrm{ab}$ & $78,04^{\mathrm{ns}}$ & 1,07 & $5,90 \mathrm{~b}$ & $91,96 \mathrm{ab}$ & $77,53 \mathrm{ab}$ & $38,30 \mathrm{ab}$ & $4,0 \mathrm{bc}$ & $15,1 b c$ & $3,7 b$ & $26,9 b c$ & $42,91 \mathrm{c}$ & $13,67 \mathrm{c}$ & $84,87 \mathrm{a}$ \\
\hline $\mathrm{CV}(\%)$ & 34,47 & 10,38 & 10,95 & 10,45 & 12,17 & 26,35 & 48,66 & 21,31 & 1,84 & 1,09 & 1,48 & 6,07 & 6,08 & 29,91 & 6,83 \\
\hline
\end{tabular}

Médias seguidas pela mesma letra não são significativamente diferentes a $0,05 \%$ de probabilidade de erro.

* significante a $0,01 \%$ e expressa em ácido cítrico $(\mathrm{g}) 100 \mathrm{~g}^{-1}$ de polpa; ** expresso em ácido ascórbico $(\mathrm{mg}) 100 \mathrm{~g}^{-1}$ de massa fresca da polpa. 
et al. (2008), em frutos de maracujá amarelo. FREIRE et al. (2010) observou diâmetros equatoriais ou transversais dos frutos de maracujazeiro amarelo variando de $61,0 \mathrm{~mm}$ a $70,3 \mathrm{~mm}$, sendo o maior valor observado com o uso de água não salina, biofertilizante bovino e com cobertura morta.

O comprimento dos frutos variou de 75,29 a $85,88 \mathrm{~mm}$ (Tabela 1). Esses valores ficaram próximos à variação nos comprimentos dos frutos de $77,0 \mathrm{a} 81,1 \mathrm{~mm}$, observada por FREIRE et al. (2010).

A relação entre o comprimento e o diâmetro dos frutos (CF/DF) é utilizada para avaliar o formato deles, considerando-se valor igual a 1 para frutos redondos e maiores para frutos ovalados (FORTALEZA et al. 2005). Desta forma, a relação CF/DF foi superior a 1 nos frutos de todas as plantas mutantes, sendo, portanto, os frutos essencialmente oblongos ou ovalados. As características do formato dos frutos são requisitadas para as indústrias de processamento, que preferem frutos oblongos, por apresentarem cerca de $10 \%$ a mais de suco do que os redondos (VIANNASILVAet al., 2010).

Os valores de espessura de casca foram elevados quando comparados aos valores obtidos por outros autores (Tabela 1). SANTOS et al. (2009) observaram em progênies de maracujazeiro amarelo espessuras de casca de $3,54 \mathrm{~mm}$ e $3,24 \mathrm{~mm}$. No mercado de fruto in natura, a espessura da casca é um fator importante a ser observado para a classificação do fruto por ser inversamente proporcional ao rendimento de suco (FERREIRA et al., 2010). O menor valor de espessura de casca observado nos frutos das plantas mutantes de maracujazeiro foi de $5,90 \mathrm{~mm}$ (Tabela 1), sendo necessária a seleção visando à redução na espessura da casca. A elevada espessura na casca pode ter sido causada pelo efeito do tratamento com raios gama.

Os valores de rendimento de suco observados variaram de $29,17 \%$ a 41,37 , destacandose os mutantes 10 e 23 pelos elevados valores observados para este parâmetro $(41,37 \%$ e $40,14 \%$, respectivamente) (Tabela 1). Esses valores foram próximos àqueles observados por SANTOS et al. (2009), que observaram valores de rendimento de suco em seleções de maracujazeiro próximos de $40 \%$.

Para a coloração da polpa dos frutos, observaram-se diferenças significativas entre os mutantes. Para valores de L, que correspondem à luminosidade, os frutos do mutante 318 apresentaram o maior valor numérico $(51,42)$. O croma ficou entre 13,67 a 27,85, indicando que a saturação ou a quantidade de pigmento na polpa é baixa. O ângulo hue para todos os genótipos ficou situado dentro do primeiro quadrante (de zero a $90^{\circ}$ ), ou seja, cor vermelha à amarela, com valores 73,90 a 84,87 , correspondendo aos mutantes 19, determinando maior intensidade de vermelho, e ao mutante 3121 , tendendo mais para a coloração amarela (tabela 1).

Considerando os atributos físicos dos frutos, destacam-se os mutantes 23 e 3121 pelo alto teor de rendimento de suco, tamanho do fruto e menor espessura da casca. O mutante 10 , apesar de apresentar fruto com elevada massa $(202,06 \mathrm{~g})$, apresentam espessura de casca bastante elevada $(8,32 \mathrm{~mm})$.

A acidez titulável e sólidos solúveis variaram de 3,5 a 4,4 e 13,1 a 15,5, respectivamente (Tabela 1). Com relação ao balanço entre açúcares e ácidos (sólidos solúveis/acidez titulável), o valor mínimo obtido foi de 3,2 e o máximo de 4,1(Tabela 1). Para a indústria de sucos de maracujá, o teor de sólidos solúveis totais de 11,4 a $15,3^{\circ}$ Brix, acidez total titulável de 2,7 a $3,9 \mathrm{~g}$ de ácido cítrico $100 \mathrm{~mL}^{-1}$ e relação Brix/acidez de 3,5 a 4,7, conferem maior palatabilidade (NAGATO et al., 2003). Comparando esses valores com os resultados obtidos neste estudo, verificou-se que os frutos provenientes das plantas mutantes apresentam valores dentro dos padrões exigidos pela indústria de sucos, evidenciando o potencial do seu cultivo.

Os valores de vitamina $\mathrm{C}$ observados nos frutos de todas as plantas mutantes ultrapassaram o valor de $20 \mathrm{mg}$ de ácido ascórbico $100 \mathrm{~g}^{-1}$ de polpa, que, segundo SANTOS et al. (2009), é normalmente observado em frutos de maracujazeiro amarelo (Tabela 1). Nesse sentido, os mutantes 24 e 318 destacaram-se

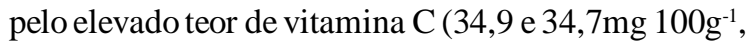
respectivamente) (Tabela 1 ).

Os frutos avaliados apresentaram características apropriadas para consumo in natura, bem como para a indústria de sucos, com destaque ao teor de vitamina C. Porém, será necessário o melhoramento genético das plantas para a redução da espessura da casca dos frutos, tendo em vista os altos valores observados para esse parâmetro.

\section{AGRADECIMENTO}

À Fundação de Amparo à Pesquisa do estado de Minas Gerais (FAPEMIG), pelo apoio financeiro.

\section{REFERÊNCIAS}

AL-QURAINY, F.; KHAN, S. Mutagenic effects of sodium azide and its application in crop improvement. World Applied Sciences Journal, v.6, n.12, p.1589-1601, 2009. Disponível em: <http://www.idosi.org/wasj/wasj6\%2812\%29/1.pdf>. Acesso em: 10 jul. 2011. ISSN: 1818-4952.

ASSOCIATION OF OFFICIAL ANALYTICAL CHEMISTS (AOAC). Official methods of analysis of the association of official analytical chemistry. 16.ed. Washington, 1997. V.2, p.37-10, 42-2, 44-3, 45-16. 
CAVICHIOLF, J.C. et al. Caracterização físico-química de frutos de maracujazeiro amarelo submetidos à iluminação artificial, irrigação e sombreamento. Revista Brasileira de Fruticultura, v.30, n.3, p.649-656, 2008. Disponível em: <http://www.scielo.br/ scielo.php?script $=$ sci_arttext\&pid $=$ S0100-2945200800030 0015\& lng=PT\&nrm=isso\&tlng=pt>. Acesso em: 27 abr. 2011. doi: 10.1590/S0100-29452008000300015.

FERREIRA, F.M. et al. Formação de super-caracteres para seleção de famílias de maracujazeiro amarelo. Acta Scientiarum, v.2, n.32, p.247-254, 2010. Disponível em: <http://periodicos.uem.br/ojs/index.php/ActaSciAgron/article/ viewArticle/3328>. Acesso em: 10 jul. 2011. doi: 10.4025/ actasciagron.v32i 2.3328 .

FORTALEZA J.M. et al. Características físicas e químicas em nove genótipos de maracujá azedo cultivado sob três níveis de adubação potássica. Revista Brasileira de Fruticultura, v.27, n.1, p.124-127, 2005. Disponível em: <http:// www.scielo.br/scielo.php? script $=$ sci_arttext $\&$ pid $=S 0100$ $9452005000100033 \& \operatorname{lng}=\mathrm{pt} \& \mathrm{nrm}=\mathrm{iso} \& \mathrm{t} \operatorname{lng}=\mathrm{pt}>$. Acesso em: 27 abr. 2011. doi: 10.1590/S0100-29452005000100033.

FREIRE, J.L. de O. et al. Atributos qualitativos do maracujá amarelo produzido com água salina, biofertilizante e cobertura morta no solo. Revista Brasileira de Ciências Agrárias, v.1, n.5, p.102110, 2010. Disponível em: <http://agraria.pro.br/sistema/ index.php?journal $=$ agraria $\&$ page $=$ article $\&$ op $=$ viewPDFInterstiti
al\&path\%5B \%5D=agraria_v5i1 a674\&path $\% 5 \mathrm{~B} \% 5 \mathrm{D}=640>$ > Acesso em: 10 jul. 2011. doi:10.5039/agraria.v5i1a674.

NAGATO, L.A. et al. Parâmetros físicos e químicos e aceitabilidade sensorial de sucos de frutas integrais, maracujá e uva, de diferentes marcas comerciais brasileiras. Brazilian Journal of Food Technology, v.1, n. 6, p.127-136, 2003.

RODRIGUES, A.C. et al. Caracterização de frutos de maracujazeiro amarelo em solo tratado com biofertilizante supermagro e potássio. Magistra, v.3, n.20, p.264-272, 2008. Disponível em: <http://www.magistra.ufrb.edu.br/publica/20.3\%20PDF/ 08_Caracterizacao_de_frutos_de_maracujazeiro>. pdf. Acesso em: 10 jul. 2011. ISSN 0102-5333.

SANTOS, C.E.M. et al. Características físicas do maracujá-azedo em função do genótipo e massa do fruto. Revista Brasileira de Fruticultura, v.31, n.4, p.1102-1110, 2009. Disponível em: $<$ http://www.scielo.br/scielo.php?script=sci_arttext\&pid=S0100$29452009000400025 \& \operatorname{lng}=\mathrm{pt} \& \mathrm{nrm}=\mathrm{iso} \& \mathrm{t} \operatorname{lng}=\mathrm{pt}>$. Acesso em: 27 abr. 2011. doi: 10.1590/S0100-29452009000400025.

VIANNA-SILVA, T. et al. Determinação da maturidade fisiológica de frutos de maracujazeiro amarelo colhidos na região norte do estado do Rio de Janeiro, Brasil. Revista Brasileira de Fruticultura, v.32, n.1, p.057-066, 2010. Disponível em: <http://www.scielo.br/scielo.php?script=sci_arttext\&pid=S0100$29452010000100009 \& \operatorname{lng}=p t \& n r m=i s o>$. Acesso em: 10 jul. 2011. doi: $10.1590 / \mathrm{S} 0100-29452010005000012$. 